\title{
Colesterol y actividad física en pandemia del COVID 19 en estudiantes del IV ciclo de Obstetricia de la Universidad Roosevelt de Huancayo. 2021
}

Cholesterol and physical activity in the Covid-19 pandemic in students of the IV cycle of Obstetrics at Roosevelt University

\author{
María Del Carmen Pilco Villagra ${ }^{1, a}$, Darwin Rivas Alarcón 1,b
}

\section{RESUMEN}

Introducción: El colesterol es considerado como el principal esteroide del ser humano, el colesterol no es dañino porque es una molécula que participa en procesos vitales para el ser humano, empero un aumento excesivo de los niveles de colesterol puede resultar perjudicial para la salud especialmente en pandemia del COVID 19, a causa del sedentarismo y el cambio de hábitos alimentarios. Objetivo: Determinar la correlación que existe entre los niveles de colesterol y la actividad física en pandemia Covid-19 en estudiantes del IV ciclo de Obstetricia de la Universidad Roosevelt. Huancayo 2021. Material y Métodos: Se realizó mediante la técnica de encuesta utilizando el cuestionario, análisis bioquímico de colesterol; para el procesamiento se usó el excel y el programa SPSS versión 23, estudio de tipo correlacional. Resultados: Los resultados obtenidos en una muestra de 36 estudiantes, por Rho de Spearman es de 0,645, significa que existe una correlación moderada, entre las dos variables con una relación considerable entre el colesterol y la actividad física en pandemia Covid-19. Conclusiones: El estudio muestra que los niveles de colesterol encontrados al análisis bioquímico y la actividad fisica tienen correlación moderada, coincidentemente con la Organización Mundial de la Salud la importancia de la actividad física para mejorar las condiciones de vida con niveles de colesterol adecuados que reflejan además la calidad de la alimentación. La inactividad física es uno de los principales problemas y el confinamiento profundiza riesgo cardio vascular por elevado colesterol, obesidad, depresión, varios tipos de cáncer, etc.

PALABRAS CLAVE: Colesterol, actividad física.

\section{SUMMARY}

Introduction: Cholesterol is considered the main human steroid, highlighting that cholesterol is not harmful because it is a molecule that participates in vital processes for humans, however an excessive increase in cholesterol levels can be detrimental to health, especially in the COVID 19 pandemic due to sedentary lifestyle and change in eating 
habits. Objective: To determine the consequence that exists between cholesterol levels and physical activity in the Covid-19 pandemic in students of the IV cycle of Obstetrics at Roosevelt University, Huancayo 2021. Material and Methods: It was carried out through the survey technique using the questionnaire, biochemical analysis of cholesterol; Excel and the SPSS version 23 program were used for processing, correlational study. Results: The results obtained in a sample of 36 students, by Spearman's Rho is 0.645 , which means that there is a moderate influence between the two variables with a considerable relationship between cholesterol and physical activity in the Covid-19 pandemic. Conclusions: The study shows that the cholesterol levels found in the biochemical analysis and physical activity have a moderate emission, coinciding with the WHO the importance of physical activity to improve living conditions with adequate cholesterol levels that also reflect the quality of Food. Physical inactivity is one of the main problems and deep confinement of cardiovascular risk due to high cholesterol, obesity, depression, various types of cancer, etc.

KEYWORDS: Cholesterol, physical activity.

\section{INTRODUCCIÓN}

La enfermedad Covid-19, como pandemia por la Organización Mundial de la Salud (OMS) en noviembre del 2020 ya se expandió a nivel global, es considerada la mayor amenaza a la salud publica en los últimos años (1).

La OMS sugiere adecuada alimentación que favorece niveles de colesterol aceptables y la actividad física como factores claves para la salud (2); esto conlleva a hacer reflexionar a los jóvenes para tener una vida saludable y como futuros profesionales de ciencias de la salud deberían de tener hábitos alimentarios saludables y actividad física.

Entre las causas que nos orientan a desarrollar alteración del colesterol encontramos la dieta rica en grasas y carbohidratos refinados, el estilo de vida, sedentarismo y los niveles de actividad física que se realicen a diario (3).

Lamentablemente, datos recolectados a nivel mundial indican que el aislamiento social por la pandemia del COVID-19, ha tenido un drástico impacto en los niveles de actividad física de la población, es una reacción inevitable del aislamiento social y las actuales condiciones de confinamiento, sus consecuencias a corto y mediano plazo podrían ser más severas de lo que pensamos. De acuerdo a la OMS, 1,9 millones de muertes anuales pueden ser atribuidas a la inactividad física, esto se agrava en pandemia COVID-19 (4).

Frente a la fatiga pandémica mejora la salud mental y mantener un estado de salud. Por lo tanto, es necesario identificar a la población que puede estar en riesgo de condiciones de salud adversas relacionadas a la inactividad física (5).
Según el Ministerio de Salud del Perú, más del 80 $\%$ de adultos jóvenes no logran cumplir con los niveles de actividad física necesarios para su salud (6).

La actividad física regular y constante tiene considerables beneficios para la salud, en todas las edades y esta contrarresta los posibles daños provocados de índole cardiovascular (7). Actualmente el Ministerio de Salud en el Perú muestra que existe un nivel de actividad física bajo, en general menor al $10 \%$ en la edad de 15 a 69 años. Además, por la baja actividad física se ha incrementado los niveles de sobrepeso y obesidad como consecuencia de la pandemia del COVID 19 (8).

Po lo expuesto, es muy importante que la actividad física, la adecuada alimentación deben ser factores básicos para el estilo de vida saludable esto conllevará a un peso adecuado, niveles de colesterol aceptables, entre otros; por ende, la investigación presenta la correlación entre las variables niveles de colesterol y la actividad física en pandemia Covid-19 en estudiantes del IV ciclo Obstetricia de la Universidad Roosevelt. 2021; reconociendo que la Universidad de acuerdo a la ley 30220 considera la responsabilidad social, la misma que debe de responder a la educación de hábitos y estilos de vida saludables que favorecen un buen estado de salud, considerando que los estudiantes de salud deben reflejar dichas prácticas es importante tener información con el fin de fortalecer y enfocar mejoras desde la universidad hacia los estudiantes y hacia la sociedad.

\section{MATERIAL Y MÉTODOS}

El estudio de esta investigación se realizó mediante el análisis de laboratorio para los niveles de colesterol y la técnica de la encuesta que utiliza la herramienta 
cuestionario para la variable de actividad física en pandemia Covid-19. Para el procedimiento de datos fue utilizado el excel y el programa de SPSS versión 23; debido que la muestra fueron los estudiantes del IV ciclo de Obstetricia. Universidad Roosevelt 2021. Esta investigación fue basada en el método científico, no experimental cuantitativo. Son acontecimientos tal y como se observan en el entorno que han sido analizados.

Hernández, Fernández y Baptista señalan que los estudios de tipo correlacional permiten conocer la relación que existe entre dos o más categorías, conceptos o variables en un determinado contexto peculiar, estos estudios logran medir el grado de asociación entre las variables; sustentadas en hipótesis sometidas a pruebas pudiendo ser una correlación positiva cuando muestran valores altos en las variables y negativa, describiendo una correlación entre las variables cuando en un momento determinado, entendido como que la primera variable tiene efecto en la otra variable, implica el uso de dos tipos de variable los intercambios que se hacen sin participación o predominación directa, y dichas interrelaciones se observan de la misma forma que se han dado en su entorno (4).

\section{RESULTADOS}

Los resultados sobre la variable niveles de colesterol en pandemia Covid -19 se muestran en la tabla 1 , y se dilucida que $31(86,1 \%)$ de los estudiantes encuestados tienen valores de colesterol normales; por otro lado, $5(13,9 \%)$ muestran valores de colesterol altos respectivamente.

Los resultados sobre la variable actividad física en pandemia Covid -19 se muestran en la tabla 2, se dilucida que $3(8,3 \%)$ de los estudiantes encuestados realizan actividad física regular; por otro lado 17 $(47,2 \%)$ realizan actividad física adecuada, 11 $(30,6 \%)$ realizan actividad física muy adecuada respectivamente.

Los resultados sobre la sobre el tipo de actividad física se muestran en la tabla 3; observamos que 6

Tabla 1. Niveles de la variable: colesterol

\begin{tabular}{lcc}
\hline Niveles & Frecuencia & Porcentaje \\
\hline Valor normal & 31 & 86,1 \\
Valor alto & 5 & 13,9 \\
Total & 36 & 100,0 \\
\hline
\end{tabular}

$(16,7 \%)$ de estudiantes encuestados realizan actividad física leve, $23(63,9 \%)$ realizan actividad física moderada y $7(19,4 \%)$ realizan actividad intensa respectivamente.

\section{Relación entre los niveles de colesterol y actividad física en pandemia Covid-19}

Existe relación significativa entre los niveles de colesterol y actividad física en pandemia Covid-19 en estudiantes del IV ciclo de Obstetricia de la Universidad Roosevelt. Huancayo 2021.

\section{Hipótesis estadística}

Ho: No existe relación significativa entre los niveles de colesterol y actividad física en pandemia Covid-19 en estudiantes del IV ciclo de Obstetricia de la Universidad Roosevelt. Huancayo 2021.

H1: Existe relación significativa entre los niveles de colesterol y actividad física en pandemia Covid-19 en estudiantes del IV ciclo de Obstetricia de la Universidad Roosevelt. Huancayo 2021.

\section{Nivel de significancia}

El nivel de significación teórica es $\alpha=0,05$

Que corresponde a un nivel de confiabilidad del 95\%

\section{Regla de decisión}

El nivel de significancia “ $p$ ” es menor que $\alpha$, rechazar Ho.

El nivel de significancia " $p$ " no es menor que $\alpha$, no rechazar Ho

\section{Prueba estadística}

Rho de Spearman

Tabla 2. Niveles de la variable: Actividad física

\begin{tabular}{ccc}
\hline Niveles & Frecuencia & Porcentaje \\
\hline Regular & 3 & 8,3 \\
Adecuado & 17 & 47,2 \\
Muy adecuado & 11 & 30,6 \\
Total & 36 & 100,0 \\
\hline
\end{tabular}

Tabla 3. Tipo de actividad física

\begin{tabular}{ccc}
\hline Niveles & Frecuencia & Porcentaje \\
\hline Leve & 6 & 16,7 \\
Moderada & 23 & 63,9 \\
Intensa & 7 & 19,4 \\
Total & 52 & 100,0 \\
\hline
\end{tabular}




\section{Decisión estadística}

Debido a que $\mathrm{p}=0.000$ es menor que 0.05 se rechaza la Ho.

\section{Conclusión}

De acuerdo a los resultados obtenidos $($ Rho $=$ $0.645 ; p=0.000$ menor que 0.05 ), existe correlación con una relación considerable entre el colesterol y la actividad física; por lo tanto, se rechaza la hipótesis nula (tabla 4).

\section{Relación entre los niveles de colesterol y tipo de actividad física en pandemia Covid-19}

Existe relación significativa entre los niveles de colesterol y el tipo de actividad física en pandemia Covid-19 en estudiantes del IV ciclo de Obstetricia de la Universidad Roosevelt. Huancayo 2021.

\section{Hipótesis estadística}

Ho: No existe relación significativa entre los niveles de colesterol y el tipo de actividad física en pandemia Covid-19 en estudiantes del IV ciclo de Obstetricia de la Universidad Roosevelt. Huancayo 2021.

H1: Existe relación significativa entre los niveles de colesterol y el tipo de actividad física en pandemia Covid-19 en estudiantes del IV ciclo de Obstetricia de la Universidad Roosevelt. Huancayo 2021.

\section{Nivel de significancia}

El nivel de significación teórica es $\alpha=0,05$

Que corresponde a un nivel de confiabilidad del 95\%

\section{Regla de decisión}

El nivel de significancia " $p$ ” es menor que $\alpha$, rechazar Ho.

El nivel de significancia " $p$ " no es menor que $\alpha$, no rechazar Ho

Tabla 4. Correlación entre niveles de colesterol y actividad física en pandemia Covid-19

\begin{tabular}{lcccc}
\hline & & $\begin{array}{c}\text { Calidad de la } \\
\text { dieta }\end{array}$ & $\begin{array}{c}\text { Circunferencia } \\
\text { cintura }\end{array}$ \\
\hline \multirow{2}{*}{ Rho de Spearman } & Colesterol & $\begin{array}{c}\text { Coeficiente de } \\
\text { correlación } \\
\text { Sig. (bilateral) }\end{array}$ & 1,000 & $0,645^{* *}$ \\
& Actividad física & N & 36 & 0,000 \\
& $\begin{array}{c}\text { Coeficiente de } \\
\text { correlación } \\
\text { Sig. (bilateral) }\end{array}$ & $0,645^{* *}$ & 36 \\
& N & 0,000 & 1,000 \\
& & 36 & 36 \\
\hline
\end{tabular}

Fuente: Base de datos (SPSS 23)

Tabla 5. Correlación entre niveles de colesterol y tipo de actividad física en pandemia Covid-19

\begin{tabular}{|c|c|c|c|c|}
\hline & & & $\begin{array}{l}\text { Calidad de } \\
\text { la dieta }\end{array}$ & $\begin{array}{c}\text { Circunferencia } \\
\text { cintura }\end{array}$ \\
\hline \multirow{6}{*}{ Rho de Spearman } & \multirow{3}{*}{ Colesterol } & $\begin{array}{l}\text { Coeficiente de } \\
\text { correlación }\end{array}$ & 1,000 & $0,680^{* *}$ \\
\hline & & Sig. (bilateral) & & 0,000 \\
\hline & & $\mathrm{N}$ & 36 & 36 \\
\hline & \multirow{3}{*}{$\begin{array}{c}\text { Tipo de } \\
\text { actividad física }\end{array}$} & $\begin{array}{l}\text { Coeficiente de } \\
\text { correlación }\end{array}$ & $0,680^{* *}$ & 1,000 \\
\hline & & Sig. (bilateral) & 0,000 & \\
\hline & & $\mathrm{N}$ & 36 & 36 \\
\hline
\end{tabular}

Fuente: Base de datos (SPSS 23) 


\section{Prueba estadística}

Rho de Spearman

\section{Decisión estadística}

Debido a que $\mathrm{p}=0.000$ es menor que 0.05 se rechaza la Ho.

\section{Conclusión}

De acuerdo a los resultados obtenidos $(\mathrm{Rho}=0,680$; $\mathrm{p}=0,000$ menor que 0,05 ), existe correlación con una relación considerable entre los niveles de colesterol y el tipo de actividad fisica, se rechaza la hipótesis nula (tabla 5).

\section{DISCUSIÓN}

Según Hernan en su estudio sobre la actividad física regular motivada en el trabajo sobre la tensión arterial y el perfil lipídico determino resultados favorables en todas las personas que presentan actividad física de forma regular con bajos niveles de actividad física. Además, se ha reportado que se hallaba asociada a los niveles de captación máxima de oxígeno. Respecto a la variable colesterol el 86,1\% tienen adecuados niveles, el $13.9 \%$ valores elevados que se encuentran sobre los $200 \mathrm{mg} /$ día (9).

En la Región Junín, las cifras de dislipidemias han ido en aumento debido a diversos factores entre los que citamos una dieta rica en grasas o carbohidratos y la falta de actividad física, por lo que la falta de actividad física ha demostrado que puede incrementar los niveles de colesterol HDL en varones adultos estudiados (10). Las investigaciones en los antecedentes presentados nos muestran que la actividad física en adultos sedentarios puede mejorar las condiciones del perfil lipídico, que en su mayoría se encuentra alterado constituyendo cuadros de dislipidemia. Además, otros estudios nos indican que el niveles de colesterol de adultos sedentarios puede mejorar cuando se realiza actividad física. Se extrapola esta información para aplicarla en la población que se estudió. Para esta investigación se planteó que la actividad física aumentada o reducida en la población tiene relación con la presentación de sus niveles de colesterol y que una actividad física disminuida puede llevar a problemas cardiovasculares en la población.

En el trabajo de investigación se encontró que el $47,2 \%$ tiene una actividad física adecuada, el $30,6 \%$ tiene una actividad física muy adecuada y el $8,3 \%$ presenta una actividad física regular, durante la pandemia del Covid-19 estos resultados finales concuerdan con los hallados por Weyh et al., quienes encontraron que el ejercicio adecuado en jóvenes, favorece la interacción para tener un sistema inmunológico, reconocido como la defensa del organismo humano contra la infección (11).

En el trabajo de investigación se encontró que el $63,9 \%$ tiene una actividad física moderada, el 19,4\% presentan actividad física intensa y $16,7 \%$ actividad física leve; durante la pandemia del Covid-19.

En el estudio se muestra que el colesterol y la actividad física presentan una correlación moderada los resultados que se hallaron por Rho de Spearman $=0,645$. y con nivel de significancia $p=0,000$ que es menor al nivel de significancia teórica de $\alpha=0,05$ se acepta la hipótesis planteada y se afirma que existe una correlación moderada con una relación confiable entre las dos variables; entonces se concluye que existe una correlación entre los niveles de colesterol y la actividad física en pandemia Covid-19; estos resultados finales concuerdan con los hallados por Álvarez, quién determino la relación existente entre los hábitos alimentarios y actividad física (12).

\section{CONCLUSIONES}

- De acuerdo a los resultados obtenidos el 86,1\% de los estudiantes presentan valores normales de colesterol y el 13,9 \% tienen valores altos de colesterol de acuerdo al análisis bioquímico, considerando la pandemia Covid-19 (tabla 1).

- Respecto a la actividad física de acuerdo a los resultados obtenidos existe $47,2 \%$ de los estudiantes tienen actividad física adecuada y el $30,6 \%$ de los estudiantes presentan actividad fisica muy adecuada; además el 8,3\% de la muestra tiene una actividad fisica regular; la OMS resalta la importancia de la actividad física para mejorar las condiciones de vida durante el camino hacia el envejecimiento. En su informe mundial sobre el envejecimiento y la salud de 2015, este organismo afirma que la actividad física a lo largo de la vida tiene como principal beneficio aumentar la longevidad (13). La inactividad física es uno de los principales problemas de salud en la actualidad y el confinamiento profundiza aún más los riesgos que conlleva como problemas de salud cardio vascular, obesidad, depresión, varios tipos de cáncer, etc., (14).

- De acuerdo a los resultados obtenidos Rho Spearman $=0,645$; existe correlación moderada con una relación confiable entre indicador de 
colesterol y actividad física en tiempos de pandemia Covid-19; consecuentemente se rechaza la hipótesis nula (tabla 4).

\section{REFERENCIAS BIBLIOGRÁFICAS}

1. Cabezas C. Pandemia de la COVID-19: tormentas y retos. Rev Peru Med Exp Salud Publica. 2020; 37 (4). DOI: 10.17843/rpmesp.2020.374.6866

2. Federik M, Calderón C. Hábitos alimentarios y COVID. Análisis descriptivo durante el aislamiento social en Argentina. Nutr Clín Diet Hosp. 2020; 40(3). DOI: https://doi.org/10.12873/403federik

3. Vera-Ponce V, Torres-Malca J. Validación de escala de cambios en los estilos de vida en el periodo de cuarentena en una población de estudiantes universitarios de Lima, Perú. Rev Fac Med Hum. 2020;20(4):614-623. DOI 10.25176/RFMH. v20i4.3193

4. Hernández R, Fernandez C, Baptista P. Metodología de la investigación. Ciudad de Mexico: McGrawHill; 2006.p.104-105.

5. Kinoshita M, Yokote $\mathrm{K}$, Arai $\mathrm{H}$, et al. Japan Atherosclerosis Society (JAS) Guidelines for Prevention of Atherosclerotic Cardiovascular Diseases 2017. J Atheroscler Thromb. 2018; 25(9): 846-984. doi: 10.5551/jat.GL2017

6. Koh Y, Park J. Cell adhesion molecules and exercise. J Inflamm Res. 2018; 11: 297-306. doi: $10.2147 / J I R . S 170262$

7. Madani A, Alack K, Richter MJ, Krüger K. Immuneregulating effects of exercise on cigarette smokeinduced inflammation. J Inflamm Res. 2018; 11: 155167. doi: $10.2147 /$ JIR.S141149

8. Miguel P, Cruz W, González J, Cardona X, Cruz L, Hernández M. Efectos beneficiosos de cambios en la dieta y ejercicios físicos en mujeres obesas con síndrome metabólico. Panor Cuba Sal. 2014;
4(3). (Citado el 15 de agosto del 2020) Disponible en: http://revpanorama.sld.cu/index.php/panorama/ article/view/121

9. Hernan A, Marit S, Oivind S, et al. Efectos favorables de la actividad física regular motivada en el trabajo sobre la tensión arterial y el perfil lipídico. Med segur Trab. 2015;61: 162-171. DOI: 10.4321/ S0465-546X2015000200003

10. Miguel PE, Peña I, Niño S, Cruz W, Niño A, Ponce D. Ensayo clínico aleatorio: papel de la dieta y ejercicios físicos en mujeres con síndrome metabólico. Aten Prim. 2012; 44(7):387-393. (Citado el 15 de agosto del 2020) Disponible en: http:// www.sciencedirect.com/science/article/pii/S021265 6711004252

11. Weyh C, Kruger K, Strasser B. Physical activity and diet shape the immune system during aging. Nutrients 2020; 12 (3). DOI: 10.3390/nu12030622.

12. Álvarez F. Hábitos alimentarios y actividad física durante el confinamiento por Covid-19 en estudiantes de la facultad de ciencias - Pontificia Universidad Javeriana.TesisdeGrado. Bogota,Colombia:Pontificia Universidad Javeriana; 2020.

13. Hammami A, Harrabi B, Mohr M, Krustrup P. Physical activity and coronavirus disease 2019 (COVID-19): specific recommendations for homebased physical training. Managing Sports and Leisure.2020.DOI: 10.1080/23750472.2020.1757494

14. Jurak G, Morrison S, Leskoek B, et al. Physical activity recommendations during the coronavirus disease-2019 virus outbreak. J Sport Health Sci. 2020; 9(4): 325-327. DOI: 10.1016/j.shs.2020.05.003

Recibido: 24/07/2021

Aceptado: 19/11/2021 